DOI : $10.14746 /$ ps.2018.1.33

\title{
Challenges and Opportunities for the EU Digital Single Market, Brussels, 23 April, 2018
}

In May 2015, the European Commission adopted the EU Digital Single Market (DSM) strategy in an effort to reduce barriers to cross-border online commerce. A series of legislative actions seek to eliminate barriers that hinder companies and consumers from using the internet to sell and buy from abroad. The aim of the conference was to answer several questions: what successes have been achieved? What challenges have been encountered and what further challenges should be expected? Participants have been asked to make an assessment of the progress made toward achieving the main priorities for strengthening the digital single market, the opportunities to accelerate progress and other potential future digital services initiatives at the EU level.

The conference was divided in 4 sessions. Speakers included experts, analysts, EU representatives, politicians, researchers and professionals from the digital sector. European Commissioner for Digital Single Market and Vice President of the European Commission, Andrus Ansip participated as a special guest.

Discussions during all sessions focused on digitalisation, the process defined as a main "disruption" of todays economy. The question about nature of this disruption - whether it is positive or negative - remained open. Three main recurring issues throughout the conference have been: data policy, digital platforms and artificial intelligence.

Data policy. Discussion was centred around General Data Protection Regulation (GDPR), which was to be implemented on 25 May 2018. Regulations of the digital market adopted within the GDPR by the European Commission invoked criticism and objections about overregulation on one side, while on the other they have been presented as an important step towards creating foundations of a coherent European policy on data protection. As A. Ansip stressed, the GDPR is a major asset for the EU - as confirmed by Facebook's plans of incorporating some of its ideas into company's global regulation.

From the scientific point of view, presented by Patrick Legros (Professor of Economics, ULB University in Brussels), there is a clash between the need for trust on the side of consumers towards companies and the need for big data collection which is necessary to provide high quality services. Data sharing creates perspectives for businesses but it also creates danger of impeding fundamental privacy right of consumers. Therefore, data policy creation requires different trade-offs. His view was shared also by experts and professionals. Discussion about data protection emerged between business and consumers. Roland Doll from Deutsche Telekom argued that the position of business and consumer protection agencies does not differ substantially, as the people protected by these agencies are in fact companies' clients. Although, as he stressed, the fundamental right for privacy should not be treated as absolute. Considering the dynamics of the process of digitalisation, a more flexible attitude is necessary, enabling to respect the right to privacy and at the same time to develop services of better quality by the companies. David Martin Luiz from the European 
Consumer Organization (BEUC) agreed with R. Doll to some extent, but he also expressed support for current steps by the European Commission, which he also considered as too slow. As Maximilian Strotmann from the Cabinet of A. Ansip pointed out, also GDPR relates to the issues of communication privacy and data protection.

Within the assessment of regulations adopted by the European Commission in the GDPR, most experts stressed the need to keep new legislation proportionate. Adina Claici from Copenhagen Economics claimed that new law should have social purpose and should not be a response to the business demands. According to Siada El Ramly from EDiMA, in current action by the Commission the political will has overtaken the actual need for regulation. Just because a new business model is created does not mean the necessity for new legislation. She described current analysis as not enough fact based and the legislation process as too slow and not keeping up with dynamic changes. The criticism of European Commission's action was shared also by business representatives: Christian Borggreen from CCIA Europe and R. Doll, who also touched upon the investment perspectives on the European market. As he claimed, the chances for investment increase in new technologies (e.g. 5G) are low due to the long term unpredictability of the environment. It is caused by EU's action as a legislator trying to regulate a diversified and competitive market.

Above criticism was rebuffed by the Commission representatives: Thomas Kramler (DSM Task Force, DG COMP) and M. Strotmann. GDPR is in their opinion a right answer to the current dynamics. It aims to replace numerous micro-regulation on the national, local and sectoral level with general European framework for all companies and customers; it will therefore limit current overregulation. Within the discussion a distinction between overregulation on one side and self-regulation on the other was evident. An interesting motion of a middle-option came up, described as co-regulation.

Majority of the speakers agreed on a positive assessment of DSM strategy and the direction of the action by the European Commission. Most important achievements have been named, among them: end of roaming charges, portability rules and limiting of geoblocking. Simultaneously the process was perceived as too slow. As MEP Eva Mydell pointed out, the discussion about digitalisation should be less politically motivated and emotional and to higher extent based on expertise and deeper understanding of modern topics such as data policy, innovations or artificial intelligence.

Platforms. State of the European digital platforms sector has been analysed as well as its competiveness and perspectives for ensuring a fair and innovation-friendly platform economy. As highlighted by S. El Ramly, what is crucial is not trying to come up with a European competitor for Google or Amazon, but to enable small and medium companies on the EU market to widen their reach and strengthen their position, as it should fuel growth in European economy. Example of Spotify has been evoked - the only European company among the TOP 25 digital platforms worldwide, which was able to secure its position despite iTunes domination on the market. Another point of the discussion was the sole definition of a platform. As T. Kramler suggested, there are various business models of digital companies and Spotify is rather to be described as a distributor of music than as a typical platform.

The term of "network effect" has been introduced in the discussion by A. Claici. In her opinion, the effect of network does not only affect big platforms by creating "winner takes it all" conditions. There are also counter-examples of network effect causing small platforms' decline - as some people leave one platform, others may follow their example. 
What's more, not all platforms enjoy network effect to the same extent. It strongly impacts social networks, which is not the case for such companies as Amazon or Uber. Referring to the issue of taxation of the "digital giants," C. Borggreen considered possibility of a side effect; some European companies could be forced to hamper their development in order to avoid high charges.

Artificial intelligence. The issue of AI comes up inevitably during discussions about data. Development of this technology bases on big data analysis. During the conference, AI has been described as "a next wave in digital revolution," able to bring new global winners. As China and USA are competing for leadership in this field today, Europe struggles with serious problems such as: insufficient digital skills, adoption of digitalisation as well as productivity puzzle. Although Europe stands well in terms of research and innovation, many companies face serious difficulties in the process of practical adoption of latest technologies and translating them into productivity gains. During the summary discussion A. Ansip stated the need to demystify AI in Europe, as it is already being used and it works within many sectors also connected with everyday reality (e. g. milk production or chicken farms). As he stressed, USA and China already have huge data sets, which Europe is missing so far. A. Ansip declared possibility of achieving the level of 20 billion USD for AI technology by the EU until 2020 (USA level in 2016). Pointing at France as a good example, he noted that Europe's success in this field depends on the investment activity of all Member States.

Tomasz MOROZOWSKI 